\title{
Improvement of the information-measuring complex for diagnostics of traction power supply objects at high-speed traffic
}

\author{
Mirjalil Yakubov ${ }^{1}$, Kamolbek Turdibekov ${ }^{1}$, Absaid Sulliev ${ }^{1}$, Islom Karimov ${ }^{1}$, Saydiaziz Saydivaliyev ${ }^{1}$, and Sarvar \\ Xalikov ${ }^{1}$ \\ ${ }^{1}$ Tashkent State Transport University, 100067 Tashkent, Uzbekistan
}

\begin{abstract}
This paper considers the improvement of the information-measuring complex for diagnostics and monitoring of traction power supply equipment under high-speed traffic, which has several features associated with a multilevel hierarchical structure, spatial distribution of objects, energy modes and functional relationships. The analysis of the transmission and conversion of measuring analogue information into a digital one, considering the maximum frequency of the primary signal spectrum components according to the sampling and quantization parameters to determine the time of the signal converted into a digital form is made. Functional dependencies and an algorithm for measuring information processing, considering the multilevel structure of traction power supply, which allow increasing the reliability and accuracy of diagnosing the main objects in high-speed traffic, are obtained.
\end{abstract}

\section{Introduction}

An integral part of the information-measuring complex (IMC) for diagnosing traction power supply objects in highspeed traffic is it's mathematical, algorithmic and software. Without its development, effective operation of this complex is impossible [1-7].

Each traction power supply object differs in its design, technological and metrological parameters. In this regard, the methods of diagnostics of these objects differ.

IIC diagnosis of traction power supply objects have several features that must be considered in the design, creation and operation of its mathematical, algorithmic and software [3-5], namely:

(a) Various physical parameters that characterize the mode of operation of interconnected technological objects of substation power equipment and various elements of the contact network with complex structural and functional relationships under load;

b) power supply objects have on the one hand a spatial distribution, and on the other hand, have also technological, information and energy interaction of elements, considering the multi-level hierarchical structure, whose input and output parameters must be coordinated structurally, metrologically and with software with the superior devices;

c) high speeds cause severe limitations on the duration of information processing in automatic control of motion processes with simultaneous execution of mathematical calculations, data processing programs at given time intervals;

The existing system of processing the received measurement information, for example, for installations of the contact network, which is the most vulnerable object, has serious drawbacks, caused by a long interval between visits to their sites by personnel, which leads to a delay in the detection of damage in their work, to the low reliability of data and subjectivity of estimates, and contributes to the development of failure processes.

d) The ICP should be improved based on modern microprocessor devices;

e) The KIC must be provided with high program stability in the process of diagnostics with the use of joint monitoring methods.

\section{Methods}

Improvement of IIC diagnostics and monitoring of the technical condition of traction power supply system equipment in high-speed traffic concerning the above-mentioned features is an urgent task.

For diagnostics of substation equipment and installations, the IMS input should receive the output signals of the primary transducers, which are carriers of information on the technical condition of the objects with the numbers $i r:$ yj; $i \in \overline{1, N}$; 
$r \in \overline{\alpha, n}$; where $N$ - number of technological objects, for example, contact network supports or number of traction transformer, etc.; $a$ - measured parameter; $r$-sensor type; $n$-sensor number. Over the output signals that differ in shape and range of change $\left[\mathrm{y}_{\min }, \mathrm{y}_{\max }\right]$ are transformed in the analogue form: their normalization, unification is performed considering steady-state dynamic ranges of output quantities: 0-10 mA, 0-10 V [10].

Considering the fact that the process of modern processing in the PIC for diagnosis and control requires a discrete and digital representation, it is necessary to provide an analogue switch, which should perform two functions:

- provide switching of analogue measuring signals coming from primary transducers (current, voltage, phase angles, temperature, electrical insulation resistance, etc.) in independent parallel communication lines via independent parallel communication lines and to one line with the serial transmission of analogue signals via this line.

- implement following continuous functions $y_{j}^{i r}, i=\overline{1, N}, r=\overline{\alpha, n}, j=\overline{1, n}$, the sequence of discontinuous or discrete functions $y_{j}^{i r}, i=\overline{1, N}, r=\overline{\alpha, n}, j=\overline{1, n}$.

According to V.A. Kotelnikov's theorem, it is theoretically sufficient to choose the sampling rate equal to;

$$
f_{D}=2 f_{\max }^{*}
$$

where $f_{\text {max }}^{*}$ - is the maximum frequency in the spectrum of the rapidly varying process, considering the Fourier decomposition. However, in diagnostic and control processes, it is reasonable to use the expression [4, 5]:

where $K D$ is the numerical coefficient.

$$
f_{D}=K_{D} f_{\max }
$$

Let us point out here that to increase the reliability in the IC of the diagnosis of traction power supply installations; it is advisable to use the cyclic mode of interrogation of the primary transducers. In this case, the cycle time Tir for processing devices $i=\overline{1, N}, r=\overline{\alpha, \eta}$, can be found as:

$$
T^{i r}=\frac{n}{f_{\text {ir }}},
$$

where $n$ is the number of sensors (transducers) connected to the processing device. The sampling frequency of the output signals for the primary transducers of the processing devices is found in the form:

$$
f_{i r}=n \cdot f_{D} \text {, }
$$

where $f_{D}$ - is the sampling frequency determined by $(2) ; n$ is the number of primary transducers. With (2) and (3) we obtain:

$$
T^{i r}=\frac{1}{f_{D}}
$$

The discrete representation of the measured value must then be converted into a digital form.

It is known [5] that the value of the analogue signal is performed according to the duration of the corresponding duration of the analogue-to-digital conversion. The error value is determined by the duration of conversion. Duration of conversion $\Delta t_{\text {пр }}$, at which the increment of a primary signal with a set rate of change does not exceed a step of quantization.

Considering the highest rates of change of measured physical quantities of objects in high-speed motion, to estimate the duration of the transformation $\Delta t_{\text {пр }}$ it is possible to use the following formula $[3,4]$ :

$$
\Delta t_{\text {пр }} \equiv\left(0,4 h \cdot f_{\max }\right)^{-1} \text { сек }
$$

where $h$-number of quantization levels of the analogue-to-digital converter; $f_{\text {max }}$ - is the maximum frequency of the controlled process.

\section{Results and Discussions}

Following the above, a multilevel functional block diagram of the IIC diagnostic and control of traction power supply is shown in Fig. 1.

The proposed multistage IMS (Figure 1) is designed for technical control, diagnostics and monitoring and consists of three stages. The first stage of the IMS includes the equipment installed mainly on the poles and mounted in the equipment of the traction substation (TS) and overhead system (OS).

The second stage of the IIC consists of data collection points, automated monitoring and control systems (AMCS), an automated system for diagnostics and monitoring of traction transformers and contact network installations, as well as tile mechanics, communication and SCADA systems.

The third stage of the IIC includes a system of operational and dispatch control, an automated control system of industrial and economic activities, as well as the means and system of data transfer to the management of "Uzbekistan Temir Yullari".

Primary processing (PA) of the measured data is carried out in real-time using methods of increasing the reliability, in the simplest way of multiple repetitions of messages and using microprocessor modules following the parameters of the diagnostic and controllable values applied [4].

From the software device, the flow of digital counts $\mathcal{V}_{i r}(O), \mathcal{V}_{i r}(T), \mathcal{V}_{i r}(2 T) \ldots$ goes to the input of the digital processing device (DPC), built on composite microprocessor modules. Here $T_{\min }=T_{i r}$ - sampling cycle (period) time for incoming information messages; $T$ - period of digital readout streams, Tmin - minimum permissible value. In this case, it is necessary to specify that the rate of primary processing of information should correspond to the sampling period $T_{i r}^{*} \leq T_{i r}$ 
Let us consider methods and algorithms for the calculation of numerical characteristics in the statistical processing of measurement information by traction power supply IMS processors. In real conditions, production, technological, electrodynamics disturbances, introduced by sensors and by the test instrument itself, affect the measurement results or contain a certain uncertainty.

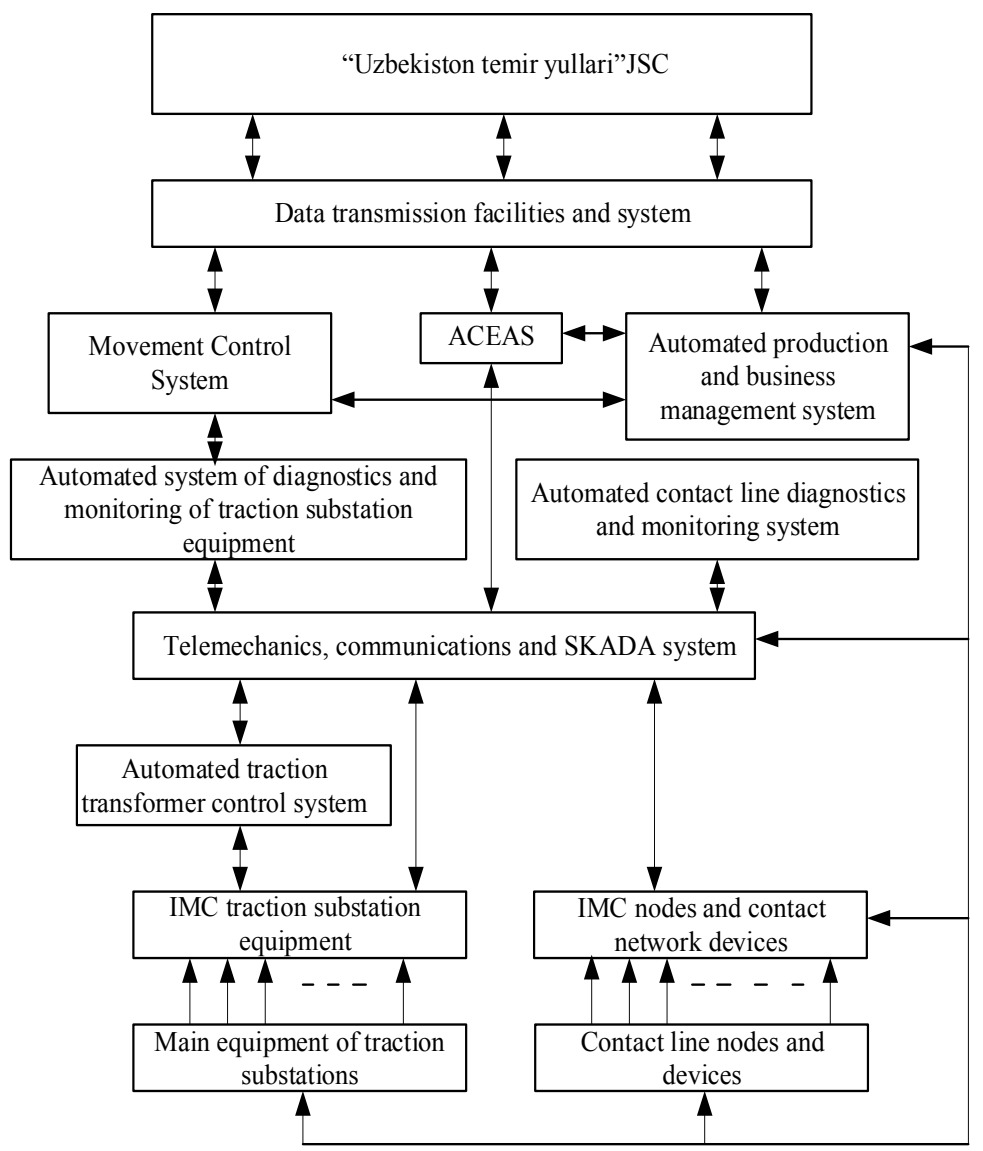

Fig. 1. Multilevel functional block diagram of IIC diagnostics and control of railroad power supply

To reduce the impact of errors or reduce the uncertainty in the results of measurements of the most important values, it is advisable to introduce methods, algorithms and programs for solving problems of mathematical statistics into the functional IMS, allowing to represent the set of measurement results of diagnostic features of technological objects in a convenient way for analysis and solving problems of diagnosis and management. These features allow you to isolate significant information from the set of data, presenting it in the form of summary indicators of diagnosis.

One of the characteristic features of traction power supply is the need to conduct statistical processing of measurement results of the most important quantities with sufficiently high accuracy and in real-time. But at the same time, statistical processing of measurements makes it possible to determine trends of changes in individual values, namely: the complex state of technological objects. For example, the state of the traction transformer of the high-speed section is characterized by the results of measurements of values of several diagnostic and control parameters (temperature and electrical state of windings, absorption coefficient, etc.) [8].

Consider the technical condition and diagnosis of traction transformers (CTs) installed on the section of high-speed traffic with numbers $i r, i \in \overline{1, N} ; r \in \alpha, n$. Let us also give a list of measured parameters with numbers $j, j \in \overline{1, n}$. In a considered IMS after discretization and quantization, a stream of measurement results in the form of a sequence of digital messages is formed. Then a cyclic interrogation with time interval Tmin $=$ Tir $=\Delta t$ is performed.

Each measured value $j$ is repeated three times, numbered from 1 to $\zeta$. To simplify further descriptions, we omit the indices $i$ and $r$. Then we obtain the series yj $1, \mathrm{yj} 2, \ldots, \mathrm{yj} \zeta$, i.e. the series yiq, $\mathrm{q} \in \overline{1, \zeta}-$ is the number of measurements of parameter $j$ at the time interval $\tau$. In practice, the measured process yjq is random. 
Consider the methodology of determining the diagnostic parameters and technical condition of CTs of traction substation, implemented by IIC, which makes measurements of the determinants of the following quantities:

$U A B, U B C$ - input and output voltages of CT, V;

$I 1, I 2$ - currents at the input and output of CT, kA;

$t$ is the temperature of the output winding;

$P 1$ and $P 2$ are the active powers at the input and output of the CT.

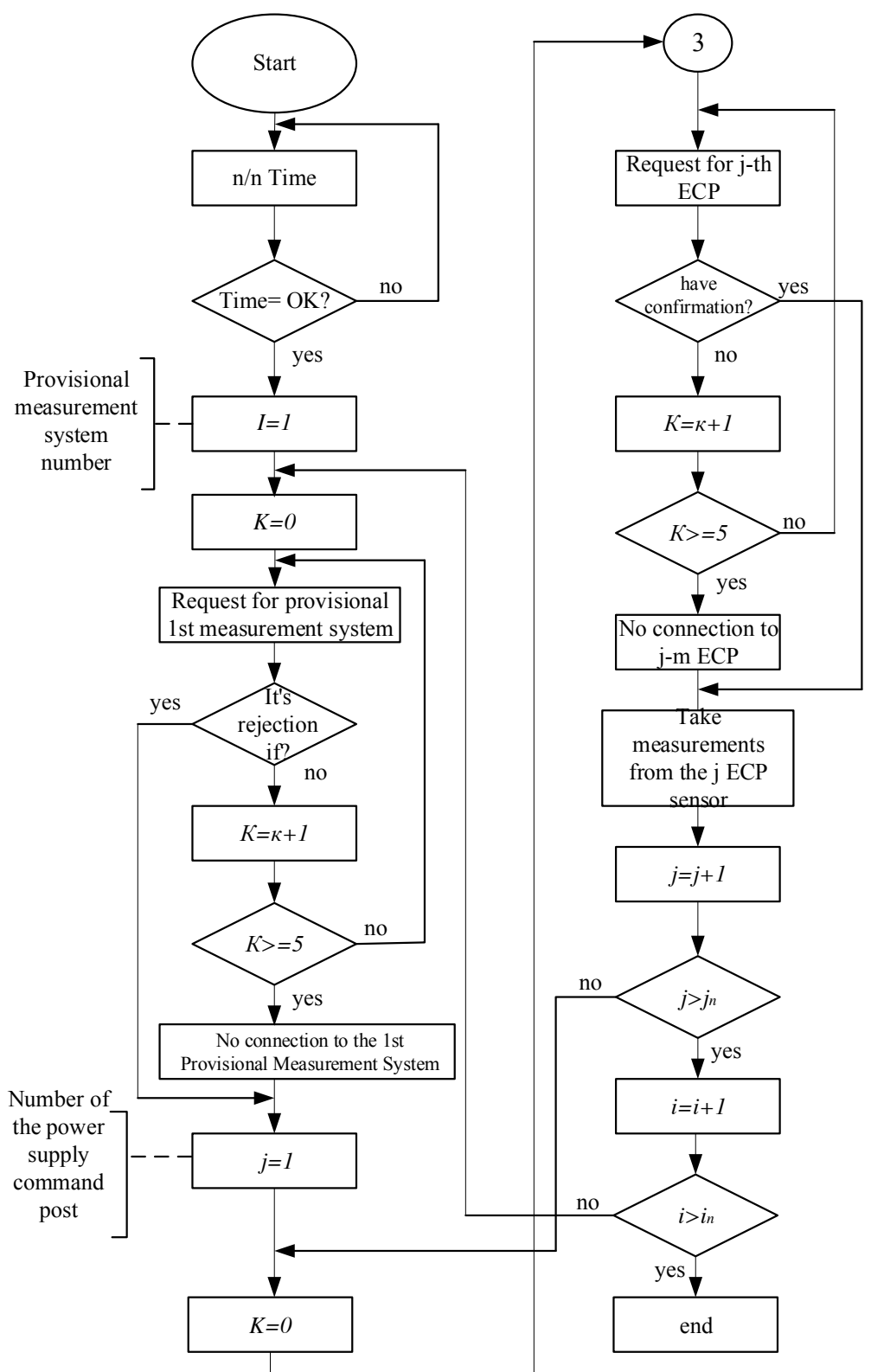

Fig. 2. Algorithm of control survey of technical condition and diagnostics of contact network installations

The reverse-sequence current, the magnitude and initial phase of which are determined by the expressions [3] are determined: 


$$
\begin{gathered}
I_{\text {об }}=\frac{\sqrt{3}}{3 U_{\text {ном }}} \cdot \sqrt{S_{A B}^{2}+S_{B C}^{2}-S_{A B} \cdot S_{B C}} ; \\
\varphi_{\text {об }}=\operatorname{arctg} \frac{\sqrt{3}}{3} \frac{S_{A B}+S_{B C}}{S_{A B}-S_{B C}}
\end{gathered}
$$

Insulation resistance of CT winding inputs, condition of magnetic wire, R60/R15 absorption coefficient, the electrical load acting on the temperature of CT upper oil layers tnom $=35-390 \mathrm{C}$ and decrease of R60/R15 ratio, up to 1,3 Mohm, are the determinants for decision making about CT operation. Voltages of 3, 5, 7 harmonics are usually equal relative to the first harmonic U5 $=6,7 \%$; $33=5,8 \%$; and $\mathrm{U} 7=4,9 \%$.

The overheating of CT windings and magnetic core is significantly affected by the increased reactive power $Q$ and the presence of higher harmonics in the power supply system, caused by the inductive nature and asymmetry of the traction load.

The main factor in assessing the technical condition is also the value of the electrical load, determined by the weight and frequency of passing trains [10].

To describe and analyze the functional dependencies, it is assumed that one-dimensional random processes $y_{j q}$, , $j \in \overline{1, n}$, are observed $q \in \overline{1, \zeta}$ for the considered CTs. For the effective solution of operational problems in realtime, let's assume that the probability of distribution of random values of CT voltage ( $y_{j q} q=$ const $)$ obeys the normal Gaussian law, which is confirmed by observations of CT operation [8].

It is known that quantitative estimates of random variables are the mathematical expectation of the output voltage $u_{j}$ in the time interval, the value of the arithmetic mean of all $u_{j}$ in the time interval, the variance and the correlation [8].

To estimate the mathematical expectation $M\left[u_{j}\right]$ we can use the arithmetic mean of the measured voltages in the time interval $\tau$ :

$$
\overline{u_{j}}=\frac{1}{\zeta} \sum_{j=1}^{\zeta} u_{j q}
$$

where $\zeta$ is the number of voltage measurements $u_{j}$ on the interval $\tau$ ?

We will take the dispersion $u_{j}$ as the dispersion in the form:

$$
S_{y i}^{2}=\frac{1}{n-1} \sum_{i=1}^{\zeta}\left(u_{j q}-u_{i}\right)^{2}
$$

Thus, on the time interval $\tau$, during which the output voltage of the CT was measured once $\zeta$, the PIC determines and stores four parameters: $\overline{u_{j}}$ - mathematical expectation; $S_{y i}^{2}$-variance, and $u_{j \min } u_{j \max }$ on the interval $\tau$.

The proposed algorithm for interrogating sensors and secondary transducers installed on the overhead system is shown in Fig. 2. Where the following designations are used:

The number of the support is denoted by the letter $i(i=1,2,3,4 \ldots)$, the number of the sensor installed on the support is denoted by the letter $j(j=1,2,3,4 \ldots)$.

Consider the proposed algorithm for interrogating signals to carry out diagnosis and monitoring of a particular section of the contact system and detection of emerging changes in its elements in a continuous automated mode.

The parameters to be diagnosed are: tension force and temperature of contact wires and carrying cables, movement of compensating device weights, inclination angles, values of pushing back on contact wire [9]. Each sensor is connected by a wire link to the nearby hub of the information gathering and transmission unit, which has a microprocessor device for primary analogue-digital processing.

Based on the developed mathematical dependencies and algorithms, the software of the information and control system for diagnostics and monitoring of traction power supply objects, which provides automation of measurement processes, collection, transmission, processing of information in real-time, was implemented.

\section{Conclusions}

The efficiency of the traction power supply system in high-speed traffic depends on the level of integration and quality measurement in the information-measuring complex for diagnosing and the process of monitoring the technical condition of electrical equipment. The proposed IMS is a set of components of measuring tools, mathematical, algorithmic and software and decision making at all levels of traction power supply system management in high-speed railroad traffic. 
When developing multilevel diagnostic ICs, it is advisable to adapt them to the existing structure of traction power supply control systems.

\section{References}

1. V.M. Savvov, Substantiation of Traction Power Supply Parameters and Electric Rolling Stock of High-Speed Rail Lines in Russia, Candidate of Technical Sciences Dissertation, S. Petersburg (2008)

2. V.M. Savvov et al., High-speed and high-speed railway transport 1, 320 (2001)

3. Q. Li, New generation traction power supply system and its key technologies for electrified railways, J. Mod. Transport 23, 1-11 (2015)

4. A.V. Efimov, A.G. Galkin, Reliability and diagnostics of railroads, Transport, Moscow (2016)

5. O.A. Stepanskaya, The peculiarities of formation of loads of electric traction network of high-speed railroad lines, Dissertation of Candidate of Technical Sciences, St. Petersburg (2018)

6. D.B. Smirnov, Improvement of calculation methods for distributed traction power supply system, Candidate of Technical Sciences Dissertation, State University of Means of Communication, Omsk (2010)

7. S.F. Amirov, M.S. Yakubov, I.A. Karimov, A system of continuous remote diagnostics of contact network with the renewable energy source, Academy of Sciences of the Republic of Uzbekistan 2, 9-104 (2021)

8. M.S. Yakubov, K.Kh. Turdibekov, A.Kh. Sulliev, Diagnosis of solid insulation of transformers by information evaluation of indirect indicators, Izvestiya Transsiba 2(42), 97-102 (2020)

9. D.V. Efanov et al., System and Method of Continuous Monitoring of Contact Network Status, Invention patent No.2701887.RU.

10. S. Amirov, M. Yakubov, K. Turdibekov, A. Sulliev, Resource-saving maintenance and repair of the Traction transformer based on its diagnostics, Int J Advanced Science and Technology 29, 1500-1504 (2020)

11. V.A. Shabanov, M.G. Bashirov, P.A. Khlyupin, Diagnostics of the technical condition of electrical equipment of power supply systems: 2 part, Methods for diagnosing the technical condition of electrical equipment, MEI Publishing House, Moscow (2018)

12. M.S. Yakubov, Zh.S. Fayzullaev, Diagnosis of the operating mode of the traction asynchronous electric motor, Bulletin of ANRUz Problems of Informatics and Energy 4, 33-41 (2018)

13. M.S. Yakubov, Zh.S. Fayzullaev, Optimization of the periodicity of technical diagnostics of maintenance and repair of traction electrical equipment of electric locomotives, Energy and Resource Saving 6, 24-32 (2018)

14. S.F. Amirov, K.K. Zhuraeva, K.Kh. Turdibekov, O.T. Boltaev, Zh.S. Fayzullaev, Magnetoelastic force transducer, Invented patent, No. IAP 20180333 Intellectual Property Agencies of the Republic of Uzbekistan (2018)

15. M.S. Yakubov, K. Turdibekov, A. Sulliev, Resource-saving maintenance and repair of the traction transformer based on its diagnostics, Journal of Tashkent Institute of Railway Engineers 16, 54-59 (2020)

16. M.S. Yakubov, S.G. Mukhamedova, Analysis of optimal periodicity of the preventive maintenance of rail service captaining into account operational technology, European Science Review 1-2, 167-171 (2018)

17. M.S. Yakubov, Z.G. Mukhamedova, U.S. Isroilov, Zh.S. Fayzullaev, Methodological aspect of continuous monitoring of traction electrical equipment diagnostics using spectral analysis methods, Chemical Technology Control and Management 3, 67-73 (2018)

18. R.Zh. Baratov, Y.Yu. Shoyimov, A.Kh. Sulliev, Ensuring constancy in high sensitivity of electromagnetic converters of mechanical quantities used in agricultural water supply, TIIIMSKH, Tashkent (1998)

19. A. Sanbetova, A. Sulliev, Sh. Kasimov, Research on Biparametric Resonant Displacement Sensors Deviation, IOP Conf. Series Materials Science and Engineering 883, 012150 (2020)

20. A. Sanbetova et al., Research on Biparametric Resonant Displacement Sensors Deviation IOP Conf. Ser.: Mater. Sci. Eng. 883, 012150 (2020) 